\title{
Chemical composition of olive oils of the cultivar Colombaia
}

\author{
By R. Boggia ${ }^{1}$, F. Evangelisti ${ }^{1}$, N. Rossi ${ }^{2}$, P.Salvadeo ${ }^{1}$ and P. Zunin ${ }^{1 *}$ \\ ${ }^{1}$ Food and Pharmaceutical Chemistry and Technology Department, University of Genova, \\ Via Brigata Salerno (ponte), 16147 Genova, Italy (e-mail: zunin@dictfa.unige.it) \\ ${ }^{2}$ Regional Laboratory of Soils and Vegetable Products Analysis -19038 Sarzana, Italy
}

\section{RESUMEN}

Composición química de aceites de oliva de la variedad Colombaia.

En este trabajo se ha estudiado la composición química de aceites de oliva mono-varietales de la variedad Colombaia. La acidez libre, el índice de peróxidos y la absorción UV confirmaron la buena calidad de los aceites analizados. Su composición en ácidos grasos resultó bastante diferente del perfil típico de ácidos grasos de los aceites de oliva virgen de la región de Liguria, pero se mantuvo dentro de los límites establecidos por los Reglamentos EC para aceites de oliva. Por otro lado, las cantidades de $\Delta_{7}$ estigmastenol resultaron normalmente superiores al límite fijado por los Reglamentos EC, que es $0.5 \%$, y el B-sitosterol total fue inferior al $93 \%$ que es el límite mínimo. La composición en compuestos polares y de la fracción volátil confirmó las características organolépticas peculiares de estos aceites. Por tanto, la composición esterólica anómala de los aceites mono-varietales de la variedad Colombaia hace necesaria una mezcla con otros aceites. Además el uso de estos aceites para la producción de aceites "Riviera Ligure" DOP debe ser controlado también cuidadosamente porque se modifican las características finales nutricionales y sensoriales.

PALABRAS-CLAVE: Aceite de oliva - Composición química - Región de Liguria - Variedad "Colombaia".

\section{SUMMARY}

Chemical composition of olive oils of the cultivar Colombaia.

The chemical composition of monovarietal olive oils from the cultivar Colombaia was studied. Free acidity, peroxide value and UV absorbance attested to the good quality of the analyzed oils. Their fatty acid composition appeared to be quite different from the typical fatty acid profile of olive oils from Liguria but met the limits reported in the EC Regulations for olive oils. On the contrary, the amounts of $\Delta_{7}$-stigmastenol were often higher than the $0.5 \%$ limit set by EC Regulations and total ß-sitosterol was below the minimum $93 \%$ limit. The composition of polar compounds and of the volatile fraction was representative of the peculiar organoleptic character of these oils. Thus, the anomalous sterol composition of the monovarietal oils from the cultivar Colombaia calls for blending with other oils. Moreover, the use of these oils for the production of PDO oils "Riviera Ligure" must also be carefully controlled because it changes their nutritional and sensorial features.

KEY-WORDS: Chemical composition - Cultivar "Colombaia" - Liguria region - Olive oil.

\section{INTRODUCTION}

The Mediterranean coastal areas have a mild, warm climate that fully meets the climatic requirements of
Olea europaea trees, and they are thus considered an ideal habitat for their growth and development.

The Taggiasca cultivar is certainly the best known among the olive cultivars in Liguria and the extra-virgin olive oils obtained from these olives are highly valued all over the world. Nevertheless, in 1997 the EC Regulation (EC Regulation N. ${ }^{\circ}$ 123/97) on Protected Designation of Origin (PDO) for "Riviera Ligure" oils states that these oils may also be obtained from different olive cultivars: the different cultivars and their proportions are also defined for the three geographical areas singled out in this Regulation. In the central part of Liguria - i.e. the Savona province - the cultivar Taggiasca prevails, but another CV, often named Colombaia or Colombara, is at times grown together with it and provides approximately $10-15 \%$ of olive production on a cultivated surface of about 400 ha. The oils obtained from Colombaia cultivar have an intense gold yellow color, with a pale green shade, a pleasant sweet flavor and a fruity, grassy aroma, more intense than in oils from the cultivar Taggiasca. The PDO Regulation states that in this area the PDO oils "Riviera Ligure- Riviera del Ponente Savonese" should be obtained from olive groves where more than $60 \%$ of olive trees are of the Taggiasca cultivar; thus, up to $40 \%$ of olive trees of the cultivar Colombaia is accepted. In the 1980s' some authors verbally reported that the oleic acid content of virgin olive oils from cultivar. Colombaia was about $65 \%$ of the total fatty acids, an amount that is definitely lower than in oils from cultivar. Taggiasca and from the other typical cultivars of Liguria, such as Lavagnina and Razzola. Moreover, a recent study on monovarietal virgin olive oils produced in Liguria (Lanteri et al., 2003) reported that the only sample from cultivar Colombaia showed a low oleic and a high linoleic and stearic acid content, together with anomalous percentage of some sterols. In fact, the $0.81 \%$ amount of $\Delta_{7}$-stigmastenol detected was even higher than the $0.5 \%$ limit set for olive oils (EC Regulation No. 1989/03).

Since the composition of extra-virgin olive oils obtained only from Colombaia olives has not yet been studied in detail and the anomalous $\Delta_{7}$-stigmastenol content could be a serious problem 
for oil producers, several samples of these oils were collected and analyzed. Moreover, some extra-virgin olive oils obtained from Taggiasca and Colombaia olives mixed in unknown proportions were also analyzed and their fatty acid and sterol content were compared with those of the analyzed monovarietal oils.

The aim of this study was to evaluate the chemical composition of monovarietal oils from the cultivar Colombaia and its compliance with the limits set for extra-virgin olive oils (EC Regulation No. 1989/03). Moreover, since Colombaia olives may be used for the production of PDO oils "Riviera Ligure Riviera del Ponente Savonese" the possible influence of these oils on the peculiar chemical and organoleptic characters of "Riviera Ligure-Riviera del Ponente Savonese" PDO oils was also considered.

\section{EXPERIMENTAL}

\subsection{Samples}

Samples (12) of monovarietal extra-virgin olive oils from olives of cultivar Colombaia were used for the study. These oils were produced between October 2001 and November 2003. Olive fruits were picked manually and oils were extracted by evening in two different oil mills, suitable for small amounts of olives. Olives were crushed in a hammer mill; the obtained olive paste was malaxed at $\mathrm{T}<25^{\circ} \mathrm{C}$ for 30 min and, finally, either a two-phase decanter or a hydraulic press, followed by centrifugation, were used for oil separation. During the same two-year period, several oil samples were collected from producers growing both Colombaia and Taggiasca olive trees and analyzed.

\subsection{Reagents}

Solvents were analytical or HPLC grade and were purchased from Merck (Darmstadt, Germany). Syringic acid, tyrosol (4-hydroxy-phenylethanol) and $\beta$-cholestanol were supplied by Fluka. Hexanal, trans-2-hexanal, hexanol, cis-3-hexenol, trans-2hexenol and nonanal were purchased by SigmaAldrich (St. Louis, MO, USA), cis-2-hexenol and trans-2-hexenyl-acetate were supplied by Avocado (Heysham, Lancashire, England).

Since $\Delta_{7}$-stigmastenol and $\Delta_{7}$-avenasterol pure standards were not commercially available, a refined sunflower oil was used for the qualitative GC-MS identification of these sterols.

\subsection{Analytical methods}

2.3.1. Determination of free acidity, peroxide value, UV absorbance, fatty acid composition and sterol fraction composition and content. These analytical variables were determined according to the European Community (EC Regulation No. 2568/91 and 1989/03).

2.3.2. Gas-Chromatography-Mass Spectrometry (GC-MS) analysis of sterol fraction. The trimethylsilyl (TMS) ethers of the purified sterols were also analyzed by GC-MS in TIC [Total lon Current mode]. Split injection (split ratio 70:1) was performed at 310 ${ }^{\circ} \mathrm{C}$ in an HP $6890 \mathrm{GC}$ System coupled with an HP 5973 quadrupole mass spectrometer (Agilent Technologies, Palo Alto, CA ). A 30m x 0.25mm i.d. $x$ $0.25 \mu \mathrm{m}$ film thickness HP5MS fused silica capillary column (Agilent Technologies, Palo Alto, CA) was used. Both isothermal (265 and $268{ }^{\circ} \mathrm{C}$ ) and programmed temperature (from $130{ }^{\circ} \mathrm{C}$ to $275^{\circ} \mathrm{C}$, at $5{ }^{\circ} \mathrm{C} / \mathrm{min}$, holding for $20 \mathrm{~min}$ ) were used in two different conditions of controlled helium flow rate $(0.7$ and $1.0 \mathrm{~mL} / \mathrm{min}$ ). The mass spectrometer interface temperature was set at $310^{\circ} \mathrm{C}$. The temperature of the ion source was $230^{\circ} \mathrm{C}$, electron energy $70 \mathrm{eV}$ and quadrupole temperature $150^{\circ} \mathrm{C}$. The acquisition range was between 30 and 500 amu.

2.3.3. Analysis of volatile fraction. The GC-MS analysis was performed with the same GC-MS system reported above. Its coupling with the Gerstel TDS2-CIS $4^{\circledR}$ system allowed the direct extraction by thermodesorption of oil volatiles in the TDS2 module and their cryoconcentration in the CIS4 cooled injector. The employed analytical conditions have been optimized in a previous study (Zunin et al., 2004) and are summarized in Figure 1.

2.3.4. Analysis of polar compounds. Polar compounds were extracted by methanol/water $(60: 40 \mathrm{v} / \mathrm{v})$ from oils dissolved in hexane and analyzed by reverse phase HPLC in gradient. A Diode Array Detector was used for the identification and quantification of the extracted compounds. Syringic acid was used as the internal standard, and the response factor of tyrosol was used for all the

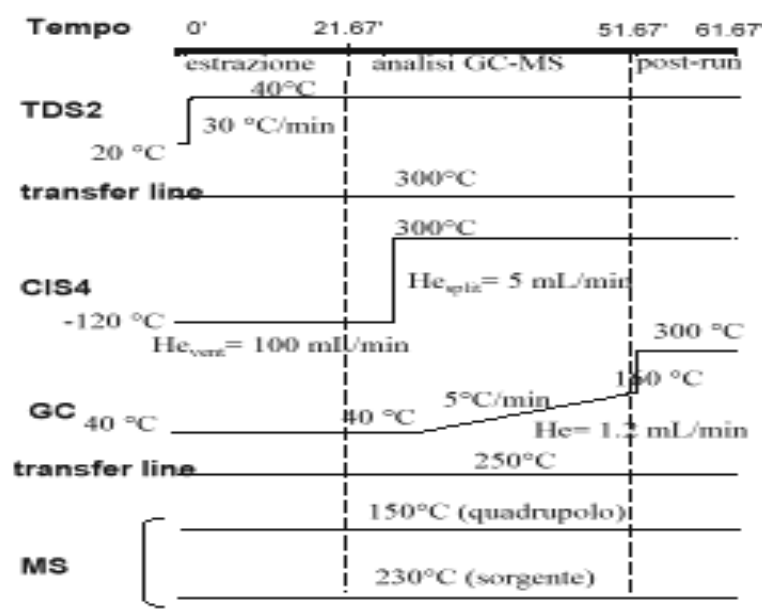

Figure 1

Analytical conditions of the TDS2 CIS4 - GC-MS system for the analysis of oil volatiles. 


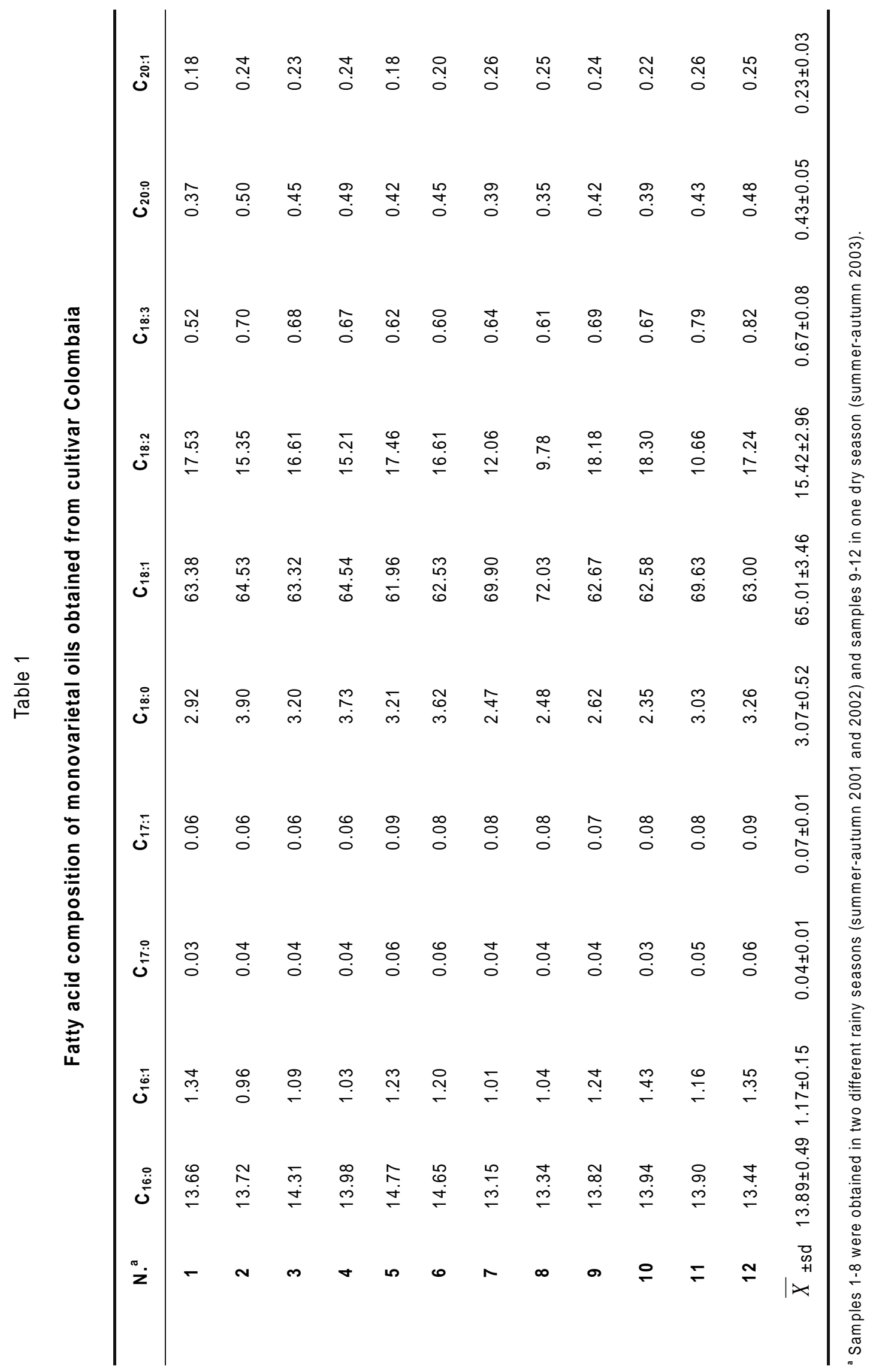


detected compounds. Therefore, the results are expressed in $\mathrm{mg} / \mathrm{Kg}$ as tyrosol. (Evangelisti et al., 1997).

\section{RESULTS}

The values of free acidity, peroxide number and UV absorbance of the 12 samples of monovarietal extra-virgin olive oils proved the good quality of the analyzed oils, since acidity was generally lower than $0.5 \%$ and peroxide value was lower than 10 . The only 3 samples with a peroxide number slightly higher than 10 showed a parallel increase in $\mathrm{K}_{232}$, thus confirming a slight increase in the development of unsaturated fatty acid oxidation.

Table 1 reports the percentage of fatty acids in monovarietal oils from cultivar Colombaia and confirms their difference from oils obtained from cultivar Taggiasca and the other most common olive cultivars of Liguria (Barichello et al., 2002). In fact, the oleic acid content was almost always markedly lower than $70 \%$, even reaching $62 \%$ while, at the same time, the content of linoleic acid often exceeded $15-16 \%$ of the total fatty acids. Such amounts of oleic and linoleic acid are often found in some Italian cultivars grown in southern Italy. Moreover, the palmitic acid content of the studied monovarietal oils was also generally higher than reported in other virgin olive oils from Liguria (Barichello et al., 1992). However, it should be emphasized that the fatty acid composition of all the analyzed monovarietal oils meets the limits set for extra-virgin olive oils (EC Regulation No. 1989/03), because fatty acid composition is used only for the evaluation of oil purity, which is guaranteed by establishing the maximum content of some "minor" fatty acids.

The analysis of the sterol fraction (EC Regulation No. 1989/03) (Figure 2) led to alarming results (Table 2 ), since the detected amounts of $\Delta_{7}$-stigmastenol were, except for one sample, higher than the $0.5 \%$ maximum limit set for all olive oils (EC Regulation No. $1989 / 03$ ) and the amounts of $\Delta_{7}$-avenasterol were often between 2 and $3 \%$ or even higher. Thus, total B-sitosterol amounts were affected by the high per cent amounts of $\Delta_{7}$-sterols and were often below the minimum $93 \%$ limit. Nevertheless, in some samples the high $\Delta_{7}$-sterol amounts were balanced by low amounts of other sterols, particularly stigmasterol, and B-sitosterol was higher than $93 \%$ and consistent with the limit reported for all olive oil categories.

With regard to the content of minor polar compounds, Table 3 reports the results obtained for the monovarietal oil samples. The amounts of total polar compounds are reported together with the amounts of the two major free phenols - tyrosol and hydroxytyrosol - and of the ester of hydroxytyrosol with the dialdehydic form of elenolic acid, which is related to a bitter taste in oils. The last column reports the sum (S) of the areas of several unknown peaks appearing in the final section of the chromatogram. This sum is particularly significant since its value is related to olive health and increases when olives were damaged by parasites or by adverse weather conditions (Evangelisti et al., 1997). The content of total polar compounds was quite variable but it often appeared high, particularly when considering that virgin olive oils from Liguria are generally rather deficient in these components. The very low $S$ amounts confirmed the good quality of the samples, but the high content of the dialdehydic form of elenolic acid linked to hydroxytyrosol might influence the oils' taste, although oils from Colombaia are generally considered sweet.

As far as the volatile fraction analysis is concerned, more than 100 compounds were detected (Figure 3). The sum of the products of lipoxygenase oxidation (LOX) pathways, which generally are the major components of the olive oil volatile fraction, ranged from 17 and $66 \%$ of the total area, with a $44 \%$ medium value and a relative standard deviation of $35 \%$. These amounts were lower than those previously reported for PDO oils "Riviera Ligure-Riviera dei Fiori", obtained in Western Liguria from $90 \%$ olives of the Taggiasca cultivar (Zunin et al., 2004). Among LOX products, the amounts of hexanal were generally higher than in those oils, while the contents of trans-2-hexenal were considerably lower. The reduction of hexanal to hexanol was favored in only some samples, and the content of C6 alcohols, both saturated and unsaturated, was quite variable. The content of
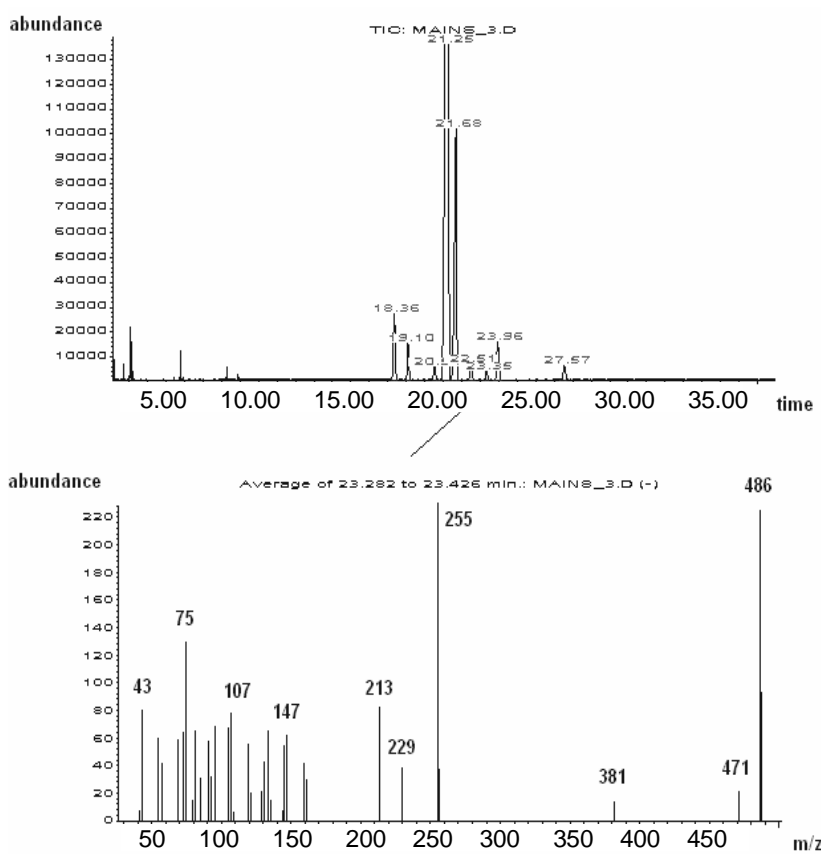

Figure 2

GC-MS plot of the sterol fraction of an extra virgin olive oil of Colombaia cultivar and mass spectrum of $\Delta 7$-stigmastenol peak. 


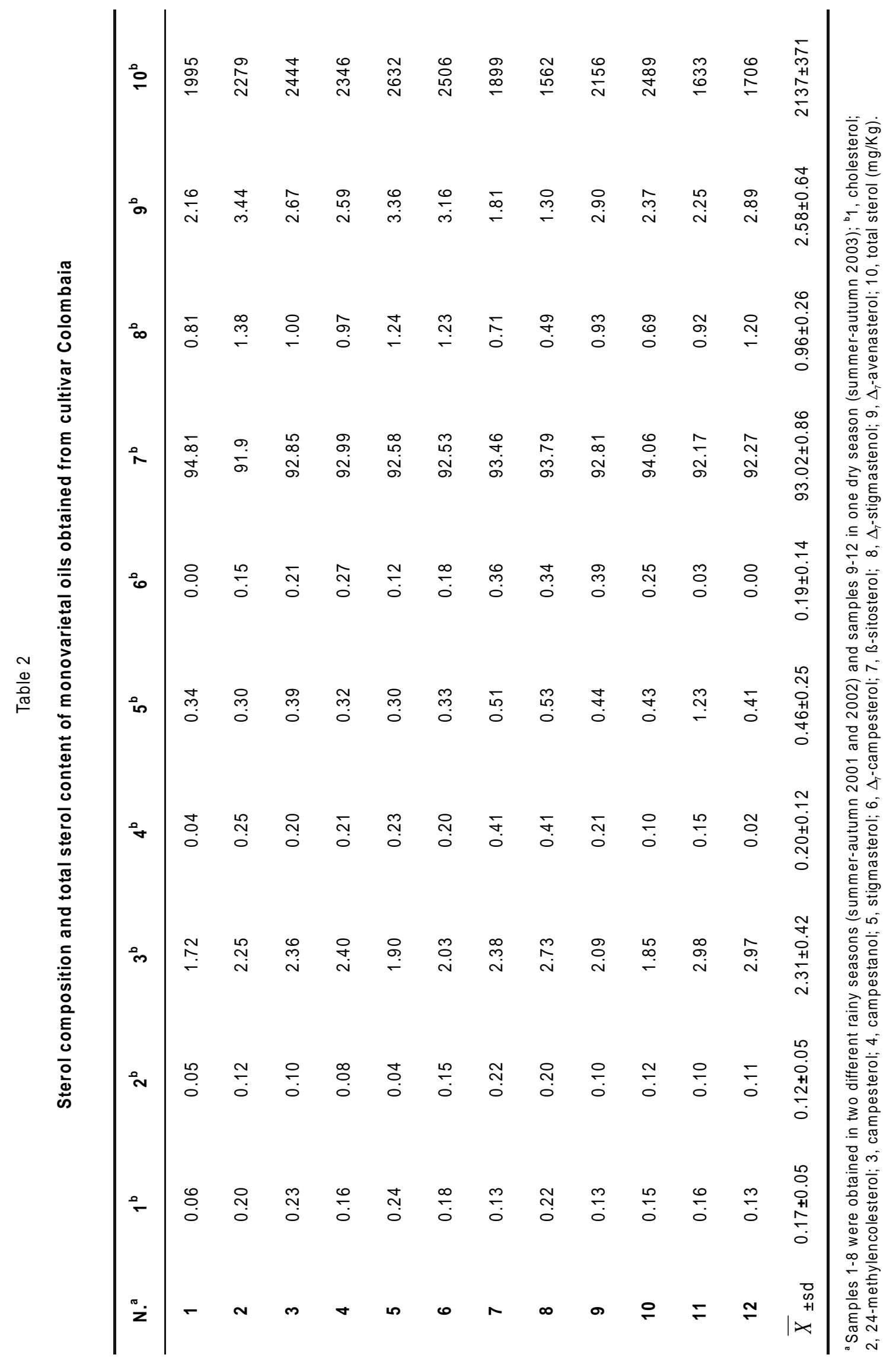


Table 3

Content of polar compounds ( $\mathrm{mg} / \mathrm{Kg}$ as tyrosol) in monovarietal oils obtained from cultivar Colombaia

\begin{tabular}{|c|c|c|c|c|c|}
\hline $\mathbf{N}^{\mathrm{a}}$ & $1^{b}$ & $2^{b}$ & $3^{b}$ & $4^{b}$ & $5^{b}$ \\
\hline 1 & 290.2 & 3.9 & 4.6 & 82.9 & 47.2 \\
\hline 2 & 318.0 & 10.4 & 2.3 & 137.1 & 25.1 \\
\hline 3 & 190.0 & 3.7 & 4.8 & 74.4 & 18.2 \\
\hline 4 & 110.8 & 2.6 & 13.0 & 9.7 & 24.0 \\
\hline 5 & 303.0 & 0.0 & 1.5 & 110.2 & 41.4 \\
\hline 6 & 262.1 & 1.4 & 2.2 & 82.0 & 40.3 \\
\hline 7 & 167.2 & 9.5 & 12.5 & 24.1 & 24.5 \\
\hline 8 & 267.0 & 5.9 & 6.7 & 58.0 & 44.0 \\
\hline 9 & 178.8 & 23.5 & 7.6 & 50.2 & 27.1 \\
\hline 10 & 117.0 & 3.0 & 4.2 & 44.9 & 19.2 \\
\hline 11 & 153.1 & 2.3 & 3.6 & 29.2 & 49.3 \\
\hline 12 & 222.3 & 2.2 & 2.4 & 46.2 & 17.6 \\
\hline $\bar{X} \pm$ sd & $215.0 \pm 72.3$ & $5.7 \pm 6.4$ & $5.5 \pm 3.9$ & $62.4 \pm 36.8$ & $31.5 \pm 12.0$ \\
\hline
\end{tabular}

cis-3-hexenylacetate was often significant. The amounts of nonanal, proposed as an index of oil oxidation, and other aldehydes, such as heptenal, heptanal, octanal, decanal and decadienal, also related to the oxidative status of the oils, were always very low and confirmed the excellent quality of the oil samples.

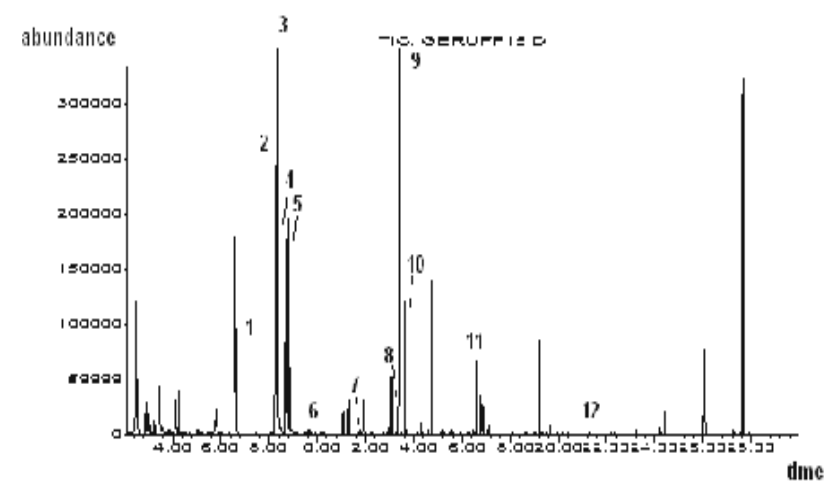

Figure 3

GC-MS plot of the volatile fraction of an extra-virgin olive oil of Colombaia cultivar. (1, hexanal; 2 , trans-2-hexenal; 3 , cis-3-hexenol; 4, trans-2-hexenol; 5, 1-hexanol; 6, heptanal; 7; trans-2-heptenal, 8; octanal; 9, cis-3-hexenylacetate; 10 , trans-2-hexenylacetate; 11 , nonanal; 12 , decenal).

\section{DISCUSSION}

The results obtained proved that the extra-virgin olive oils from the cultivar Colombaia have several specific characteristics that distinguish them from the most common olive oils from Liguria. With respect to their sensorial features, the high amounts of hexanal, often related to a sweet fruity flavor, and of cis-3-hexenylacetate, which also provides a pleasant fruity aroma (Morales et al., 1997; Kiritsakis, 1998) can be associated to the pleasant soft fruity flavor often detected by tasters of these oils. Although the rather high amount of the dialdehydic form of elenolic acid linked to hydroxytyrosol might contribute to a bitter taste, the low content of trans-2-hexenal, a coumpound often related to the bitter green component of aroma (Morales et al., 1997; Kiritsakis, 1998) might explain their overall delicate aroma. As far as the fatty acid composition is concerned, the low oleic and high linolenic acid content of oils from cultivar. Colombaia are not a serious problem, since virgin olive oils from other olive cultivars may have a similar fatty acid pattern. Nevertheless, the high oleic acid content (generally $>75 \%$ ) of extra-virgin olive oils from Liguria is one of their specific nutritional qualities, because of the important role of this acid in the Mediterranean diet. In fact, although oleic acid is 
less effective than polyunsaturated fatty acids in lowering blood cholesterol, it reaches this goal by lowering only LDL-cholesterol, while it does not lower HDL-cholesterol, which is essential for cholesterol clearing. Moreover, oleic acid is also more resistant to heat and oxidative stress than polyunsaturated fatty acids.

However, a serious problem with these oils is the anomalous composition of their sterol fraction, which is not consistent with EC Regulations and could lead to legal actions on charges of adulteration against the oils obtained exclusively or mainly from this cultivar. This risk is really serious since such anomalies have not been frequently reported. A few years ago, some authors reported that the content of $\Delta_{7}$-stigmastenol in olive oils could reach $0.8 \%$ of the total sterol content (Karleskind and Wolf, 1992). These authors also reported similar amounts of $\Delta_{7}$-avenasterol, but their quantitative evaluation could have been affected by the use of classical packed chromatographic columns. Lately, amounts of $\Delta_{7}$-stigmastenol higher than the $0.5 \%$ EC limit were detected in Spanish virgin olive oils from cultivar. Empeltre (Gracia Gómez, 2001) and these values seemed higher when rainfall was scarce and no extra watering was supplied. As far as the oils from Colombaia cultivar are concerned, it is extremely unlikely that the high content of $\Delta_{7}$-stigmastenol in the samples was a consequence of drought, since two rainy seasons (summer-autumn 2001 and 2002: samples 1-8) as well as one very dry season (summer-autumn 2003: samples 9-12) were studied and no significant differences were observed.

Thus, it was assumed that in Colombaia oils unknown compounds co-eluting with $\Delta_{7}$-sterols in the analytical conditions of the Official Method of Analysis may be present. In order to investigate this assumption, the GC-FID of the sterol fraction of Colombaia oils was supported by a GC-MS analysis. The sterol fraction of a refined sunflower oil was used for RT identification of the $\Delta_{7}$-sterols and for the acquisition of their MS spectra in the same analytical conditions. The hypothesis of a possible co-elution of $\Delta_{7}$-stigmastenol and cycloartenol, a terpenic alcohol which has similar gas-chromatographic behavior, was considered with particular attention. Nevertheless, neither isothermal nor programmed temperature analyses in different conditions of temperature and flow disclosed the presence of co-eluted peaks. Moreover, the MS spectra of the two $\Delta_{7}$-sterols were consistent with the spectra obtained from the sunflower oil analysis, and the MS spectrum of $\Delta_{7}$-stigmastenol also showed a good match (78\%) with those reported for this compound in the electronic library WILEY 275, which does not contain the MS spectrum of $\Delta_{7}$-avenasterol. The ions having $\mathrm{m} / \mathrm{z} 408,393$ and 365 , i.e. the major ions in the cycolartenol spectrum, were completely absent in the MS spectrum of the $\Delta_{7}$-stigmastenol peak, thus confirming a successful sterol fraction TLC purification.

In order to reach a preliminary evaluation of the real impact of the anomalous sterol composition of these oils on olive oil production in the Savona province, several farms growing mixed Colombaia and Taggiasca trees were singled out in this area and their olive oils were analyzed. The detected amounts of oleic acid, ranging between 69 and $79 \%$, represented a probable prevailing of oils from Taggiasca olives. Confirming this assumption, these oils were generally consistent with the sterol composition limits (EC Regulation No. 1989/03), with the exception of one sample, which had a B-sitosterol content lower than $93 \%$ and a $\Delta_{7}$-stigmastenol content close to the $0.5 \%$ limit. These findings appear quite reassuring, since they mean that the present field distribution of the cultivars generally allows "dilution" of the anomalous composition of Colombaia oils.

\section{CONCLUSIONS}

The results obtained confirm that the volatile fraction of monovarietal Colombaia oils can bring excellent organoleptic features to blended oils. Nevertheless, their fatty acid composition suggests a reduction in their employment in the production of PDO oils "Riviera Ligure - Riviera del Ponente Savonese", since it causes a decrease in their characteristic high content of oleic acid.

Finally, sterol composition is certainly a delicate matter and it makes the blending of these oils with other oils absolutely necessary: in fact, if these monovarietal oils were to be marketed as such, a random control would detect amounts of $\Delta_{7}$-stigmastenol and/or $\beta$-sitosterol that go beyond the European limits for olive oils. Although the reasons for the anomalous composition of these oils are unknown at present, olive growers should be conscious of the potential risks connected with their marketing, as well as of the need for their blending with other olive oils and for careful planning when planting new olive trees.

\section{ACKNOWLEDGEMENTS}

This research was carried out for the Regione Liguria project "Miglioramento qualitativo della produzione di olio di oliva ligure", supported by European Union (EC Regulation N. ${ }^{\circ}$ 528/99).

\section{REFERENCES}

Barichello R, Evangelisti F, Pini S, Rossi N, Zunin P. 2002. L'olio di oliva ligure. "Quaderni di Agricoltura". Publication supported by grants from the European Union (EC Regulation N. ${ }^{6}$ 528/99). Microarts Ed., Recco, Genova. 
European Community Regulation No. 2568/91. Off. J. Eur. Communities: L248 (05/09/1991).

European Community Regulation No. 123/97. Off. J. Eur. Communities: L022 (24/01/1997).

European Community Regulation No. 1989/03. Off. J. Eur. Communities: L295 (13/11/2003).

Evangelisti F, Zunin P, Tiscornia E, Petacchi R, Drava G, Lanteri S. 1997. Stability to oxidation of virgin olive oils as related to olive conditions: study of polar compounds by chemometric methods. J. Am. Oil Chem. Soc. 74, 1017-1023.

Gracia Gomez MS 2001. Composición química de distintas calidades de aceites de oliva virgen de la variedad "Empeltre" en el Bajo Aragón. Grasas y Aceites 52, 52-58

Karleskind A, Wolff JP. 1992. Manuel des Corps Gras. Ed. Technique et Documentation, Lavoisier, Paris Cedex.

Kiritsakis AK. 1998. Flavor components of olive oil - A review. J. Am. Oil Chem. Soc. 75, 673-681.
Lanteri S, Boggia R, Rossi N. 2003. Multivariate characterisation of monovarietal virgin olive oils from Liguria. V COLLOQUIUM CHEMIOMETRICUM MEDITERRANEUM, 25-27 June, Ustica, Italy.

Morales MT, Rios JJ, Aparicio R. 1997. Changes in the volatile composition of virgin olive oil during oxidation: flavors and off-flavors. J. Agric. Food Chem. 45, 2666-2673.

Zunin P, Boggia R, Lanteri S, Leardi R, De Andreis A, Evangelisti F. 2004. Direct thermal extraction and gaschromatographic-mass spectrometric determination of volatile compounds of extra-virgin olive oils. $J$. Chromatog. A 1023, 271-276. 